\title{
Efecto de la altura de medio filtrante y la velocidad de filtración en el comportamiento de distintos modelos de filtro de arena en instalaciones de riego por goteo con aguas regeneradas
}

\author{
Carles Solé-Torres ${ }^{1}$, Miquel Duran-Ros ${ }^{1}$, Gerard Arbat ${ }^{1}$, Joan Pujol ${ }^{1}$, Francisco Ramírez de \\ Cartagena ${ }^{1}$, Jaume Puig-Bargués ${ }^{1}$ \\ 1 Departamento de Ingeniería Química, Agraria y Tecnología Agroalimentaria, Escuela Politécnica \\ Superior, Universidad de Girona, C. de M. A. Capmany 61, 17003 Girona; carles.sole@udg.edu; \\ miquel.duranros@udg.edu; gerard.arbat@udg.edu; joan.pujol@udg.edu; francisco.ramirez@udg.edu; \\ jaume.puig@udg.edu
}

\begin{abstract}
Resumen: Los filtros de arena son habitualmente utilizados en las instalaciones de riego por goteo para reducir la obturación de los goteros, siendo especialmente indicados cuando se utilizan aguas residuales regeneradas, aunque poco se conoce acerca de cuáles son sus condiciones operativas que permitan garantizar una buena calidad de filtrado y un bajo consumo energético. Por este motivo, en el presente trabajo se estudió, utilizando aguas residuales regeneradas, el comportamiento de tres modelos de filtro de arena con distintos diseños de drenaje operando con dos alturas de lecho filtrante $(20$ y $30 \mathrm{~cm})$ y dos velocidades de filtración (30 y $60 \mathrm{~m} / \mathrm{h}$ ). Cada una de estas condiciones operativas se ensayó durante $250 \mathrm{~h}$, registrándose los volúmenes filtrados y de lavado, las presiones y parámetros fisicoquímicos a la entrada y a la salida de los filtros y el consumo eléctrico de la bomba de la instalación. La reducción de turbidez en el proceso de filtración se vio afectada por la velocidad de filtración, siendo mayor con $30 \mathrm{~m} / \mathrm{h}(34.2 \%)$ que con $60 \mathrm{~m} / \mathrm{h}(11.3 \%)$. El drenaje con medio poroso presentó una reducción significativamente superior de turbidez (26.3\%) respecto a los otros dos diseños, filtrando significativamente más volumen de agua por unidad de energía eléctrica consumida (8.4 m3/kWh).
\end{abstract}

Palabras clave: riego localizado, eficiencia de filtración, volumen filtrado, eficiencia energética, obturación.

\section{Introducción}

El uso de aguas residuales regeneradas en agricultura se ha convertido en una de las principales soluciones para aliviar la situación de escasez global de agua [1]. La mejor técnica para el uso de dichas aguas, desde un punto de vista sanitario y medioambiental, es el riego por goteo [2] aunque presenta un riesgo de obturación de los goteros [2, 3]. Para evitarlo, resulta imprescindible disponer de un sistema de filtrado $[4,5,6]$, aunque no siempre evita completamente la aparición de obturaciones [7]. Los filtros de arena son los que ofrecen mejor protección [8] contra el atascamiento de los goteros $[5,6]$ ya que eliminan más eficazmente los sólidos en suspensión presentes en las aguas regeneradas [7,9]. El proceso de filtración, y especialmente el de contralavado del filtro, requieren presiones de trabajo elevadas, por lo que los filtros juegan un papel importante en el consumo energético de los sistemas de riego por goteo [10], el cual se debería optimizar debido al incremento del coste de la energía [11]. 


\section{CONGRESO IBÉRICO DE AGROINGENIERÍA \\ X CONGRESSO IBÉRICO DE AGROENGENHARIA \\ 3 - 6 septiembre 2019, Huesca - España}

La mejora del diseño de los filtros de arena puede reducir el consumo de energía e incrementar la eficiencia de filtración [12]. En los filtros de arena, la mayor parte de la pérdida de carga se localiza en los elementos auxiliares como el plato difusor y el sistema de drenaje [13]. Por su parte, Bové et al. (2017) [14] determinaron que mejorando el diseño del drenaje, se podía reducir significativamente la caída de presión en los filtros.

Aunque muchos estudios relacionan las características físicas del medio filtrante con la retención de sólidos, existe una falta de información sobre cómo algunas condiciones operativas de los filtros de arena, como la altura del medio filtrante y la velocidad de filtración, influyen en el proceso de filtración. Además, la reducción de la altura del medio de filtración implicaría una reducción del impacto ambiental [15].

El objetivo del presente estudio es analizar cómo afectan la altura del medio filtrante, la velocidad de filtración y tres diseños de drenaje distintos (el propuesto por Bové et al., 2017 [14] y otros dos de comerciales) tanto en la calidad de filtración, como en el consumo energético y de agua.

\section{Materiales y métodos}

\subsection{Diseño experimental}

Se utilizó el agua residual regenerada de la Estación Depuradora de Aguas Residuales (EDAR) de Celrà (Girona).

El sistema experimental consistió en tres filtros de arena con tres drenajes distintos: un filtro modelo FA1M (Lama, Sevilla, España) con drenaje de brazos colectores, otro modelo FA-F2-188 (Regaber, Parets del Vallès, España) con drenaje de cúpulas insertadas y el filtro experimental propuesto por Bové et al. (2017) [14] con drenaje de medio granular. La Figura 1 muestra el diseño de los tres drenajes utilizados mientras que la Tabla 1 muestra sus principales características.

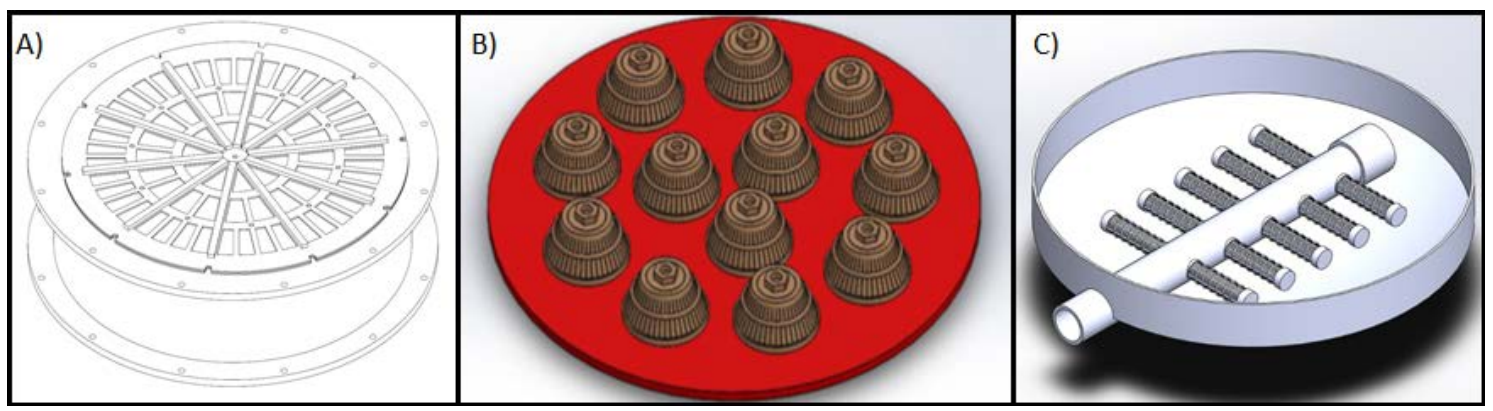

Figura 1. Diseños de drenajes utilizados: medio poroso (A), cúpulas insertadas (B) y brazos colectores (C).

Tabla 1. Características de los drenajes utilizados en el ensayo.

\begin{tabular}{ccccc}
\hline $\begin{array}{c}\text { Diseño del drenaje } \\
\text { del filtro }\end{array}$ & $\begin{array}{c}\text { Diámetro } \\
\text { nominal del } \\
\text { filtro } \\
(\mathbf{m m})\end{array}$ & $\begin{array}{c}\text { Superficie } \\
\text { de filtración } \\
\left(\mathbf{m}^{\mathbf{2}}\right)\end{array}$ & $\begin{array}{c}\text { Caudal } \\
\text { máximo } \\
\text { filtrado } \\
\left(\mathbf{m}^{3} / \mathbf{h}\right)\end{array}$ & $\begin{array}{c}\text { Altura } \\
\text { máxima del } \\
\text { lecho } \\
\text { filtrante } \\
(\mathbf{m})\end{array}$ \\
\hline Medio poroso & 500 & 0.1960 & 20 & 0.70 \\
Cúpulas insertadas & 508 & 0.2026 & 18 & 0.69 \\
Brazos colectores & 500 & 0.1960 & 23 & 0.40 \\
\hline
\end{tabular}




\section{CONGRESO IBÉRICO DE AGROINGENIERÍA \\ X CONGRESSO IBÉRICO DE AGROENGENHARIA \\ 3 - 6 septiembre 2019, Huesca - España}

Todos los filtros fueron rellenados con arena silícea con un diámetro efectivo de $0.48 \mathrm{~mm}$, coeficiente de uniformidad de 1.73 , densidad real $2454 \mathrm{~kg} / \mathrm{m}^{3}$, densidad aparente $1509 \mathrm{~kg} / \mathrm{m}^{3} \mathrm{y}$ porosidad de 0.39. Cada filtro tenía asociado una subunidad de riego, consistente en 4 laterales de $90 \mathrm{~m}$ cada uno.

El agua residual se bombeó de la estación depuradora hacia los filtros mediante un sistema de impulsión gobernado por un sistema de supervisión, control y adquisición de datos (SCADA), $\mathrm{y}$ un variador de frecuencia que ajustaba el punto de funcionamiento de la bomba. El caudal de funcionamiento se midió con un caudalímetro electromagnético, que disponía de un transmisor de impulsos con señal 4-20 mA que permitía conocer el caudal exacto de funcionamiento. El sistema experimental permitía el funcionamiento de un único filtro a la vez. Debido a que en el filtro circulaba más caudal de agua del necesario en la subunidad de riego, una válvula proporcional de tres vías conducía el caudal de agua filtrado sobrante hacia un tanque donde se almacenaba, para posteriormente ser utilizada en los contralavados de los filtros. El agua se cloró después de pasar los filtros hasta alcanzar los 2 ppm de cloro libre, y hasta los 4 ppm cuando se utilizaba para el contralavado de los mismos. Los contralavados de los filtros, de 3 minutos de duración, se activaban cuando la pérdida de carga sobrepasaba los $50 \mathrm{kPa}$.

Se instalaron sensores para medir la calidad del agua antes y después de ser filtrada. Los parámetros que se midieron antes del proceso de filtración fueron conductividad eléctrica (CE), $\mathrm{pH}$, temperatura, oxígeno disuelto (OD) y turbidez. Los parámetros que se determinaron después del proceso de filtración fueron únicamente el oxígeno disuelto y la turbidez.

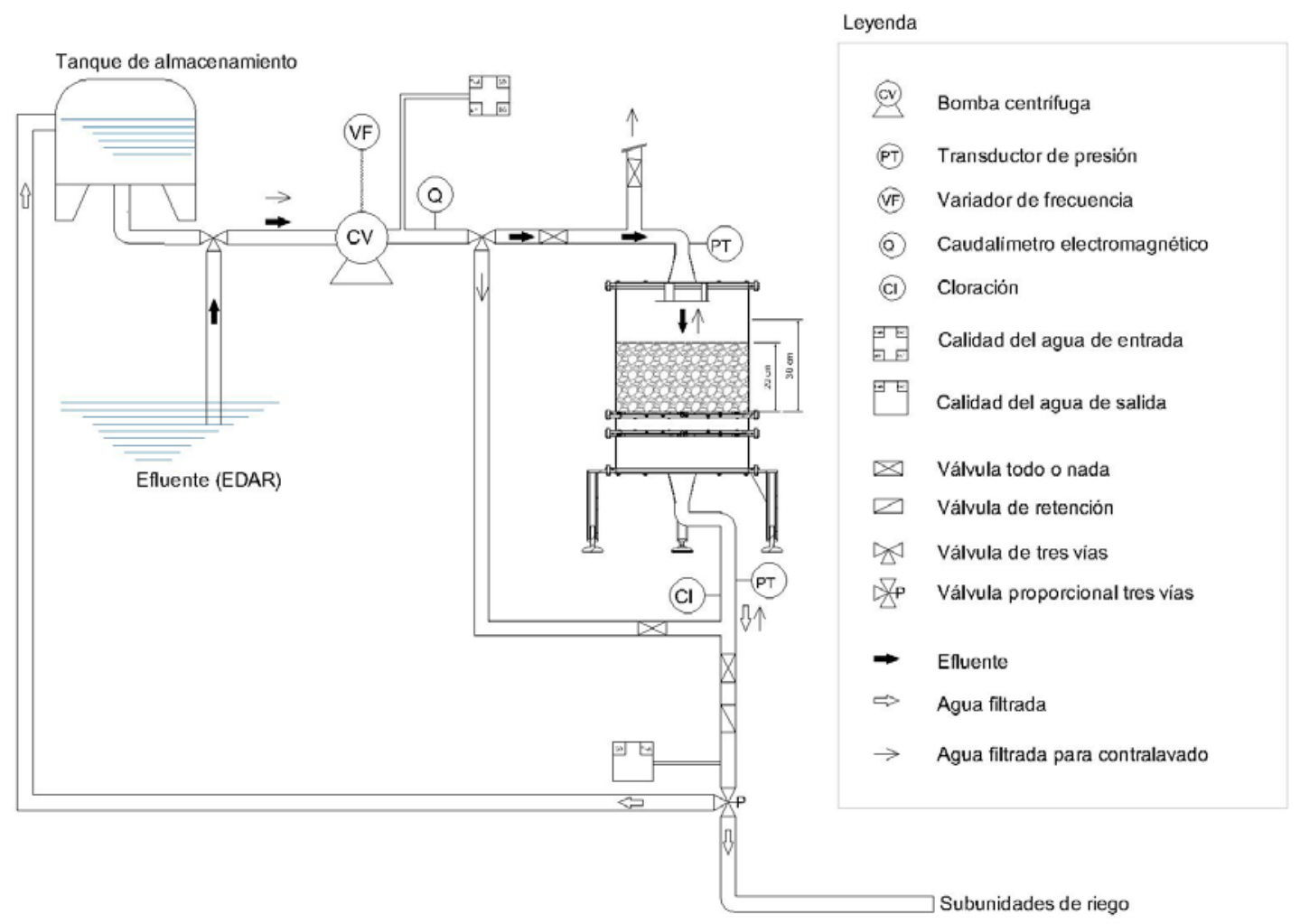

Figura 2. Esquema del diseño experimental. Para simplificar, sólo se representa un filtro. 


\section{CONGRESO IBÉRICO DE AGROINGENIERÍA \\ X CONGRESSO IBÉRICO DE AGROENGENHARIA \\ 3 - 6 septiembre 2019, Huesca - España}

\subsection{Procedimiento operacional}

Cada filtro se utilizó durante $1000 \mathrm{~h}$. Se establecieron dos sesiones diarias de riego de 4 horas por filtro cada una. A lo largo de esas $1000 \mathrm{~h}$, se ensayaron dos alturas de lecho filtrante $(20$ y 30 $\mathrm{cm}$ ) y dos velocidades de filtración (30 y $60 \mathrm{~m} / \mathrm{h}$ ), lo que supuso un total de cuatro condiciones operativas distintas. Cada condición operativa fue probada durante $250 \mathrm{~h}$ para cada filtro. Las velocidades de filtración de 30 y $60 \mathrm{~m} / \mathrm{h}$ correspondieron a caudales nominales de 6000 y $12000 \mathrm{l} / \mathrm{h}$, respectivamente, con caudales de contralavado de 9000 y 15000 l/h, respectivamente. Puesto que el sistema de supervisión, control y adquisición de datos (SCADA) implantado en la instalación [16] medía en tiempo real los niveles de turbidez del agua de entrada, se estableció que un sistema de alarma para detener el funcionamiento del sistema si se sobrepasaban las $20 \mathrm{FNU}$.

\subsection{Caracterización del agua de entrada}

Los parámetros fisicoquímicos fueron medidos y registrados cada minuto en el sistema SCADA indicado en la sección anterior. La Tabla 2 muestra los valores medios de cada parámetro del agua de entrada para cada condición operativa ensayada.

Tabla 2. Media y error estándar de los valores de entrada del efluente utilizado. Letras diferentes significan diferencias significativas $(\mathrm{P}<0.05)$ entre los valores de un mismo parámetro.

\begin{tabular}{|c|c|c|c|c|c|c|c|}
\hline $\begin{array}{c}\text { Altura } \\
\text { lecho } \\
(\mathrm{m})\end{array}$ & $\begin{array}{l}\text { Velocidad } \\
\text { de } \\
\text { filtración } \\
(\mathrm{m} / \mathrm{h})\end{array}$ & $\begin{array}{l}\text { Diseño del } \\
\text { drenaje }\end{array}$ & $\begin{array}{l}\text { Conductividad } \\
\text { eléctrica } \\
(\mathrm{dS} / \mathrm{m})\end{array}$ & $\begin{array}{c}\text { Oxígeno disuelto } \\
(\mathrm{mg} / \mathrm{l})\end{array}$ & $\begin{array}{l}\mathrm{pH} \\
(-)\end{array}$ & $\begin{array}{c}\text { Temperatura } \\
\left({ }^{\circ} \mathrm{C}\right)\end{array}$ & $\begin{array}{c}\text { Turbidez } \\
\text { (FNU) }\end{array}$ \\
\hline \multirow[t]{3}{*}{0.20} & 30 & Medio poroso & $2.68 \pm 1.24 \mathrm{abc}$ & $2.69 \pm 0.15$ def & $7.04 \pm 0.02 \mathrm{f}$ & $15.29 \pm 0.51 \mathrm{~g}$ & $8.16 \pm 0.36 \mathrm{ab}$ \\
\hline & & $\begin{array}{l}\text { Cúpulas } \\
\text { insertadas }\end{array}$ & $2.90 \pm 0.79 \mathrm{a}$ & $3.07 \pm 0.11$ cde & $7.10 \pm 0.02 \mathrm{f}$ & $15.31 \pm 0.31 \mathrm{~g}$ & $7.49 \pm 0.36 \mathrm{abc}$ \\
\hline & & Brazos colectores & $2.89 \pm 0.57 \mathrm{a}$ & $2.89 \pm 0.09 \mathrm{def}$ & $7.07 \pm 0.02 \mathrm{f}$ & $16.35 \pm 0.28 \mathrm{~g}$ & $8.51 \pm 0.42 \mathrm{a}$ \\
\hline \multirow[t]{3}{*}{0.20} & 60 & Medio poroso & $2.79 \pm 0.40 \mathrm{ab}$ & $3.44 \pm 0.08 \mathrm{bcd}$ & $7.29 \pm 0.02 \mathrm{e}$ & $21.03 \pm 0.40 \mathrm{def}$ & $5.82 \pm 0.21 \mathrm{~cd}$ \\
\hline & & $\begin{array}{l}\text { Cúpulas } \\
\text { insertadas }\end{array}$ & $2.18 \pm 0.71 \mathrm{de}$ & $4.27 \pm 0.11 \mathrm{a}$ & $7.71 \pm 0.02 \mathrm{a}$ & $24.01 \pm 0.21 \mathrm{ab}$ & $2.84 \pm 0.17 \mathrm{e}$ \\
\hline & & Brazos colectores & $2.54 \pm 0.29 \mathrm{bc}$ & $3.93 \pm 0.16 \mathrm{ab}$ & $7.52 \pm 0.02 b$ & $25.02 \pm 0.11 \mathrm{a}$ & $3.50 \pm 0.30 \mathrm{e}$ \\
\hline \multirow[t]{3}{*}{0.30} & 30 & Medio poroso & $1.85 \pm 1.49 \mathrm{e}$ & $3.37 \pm 0.28$ bcde & $7.71 \pm 0.05 \mathrm{a}$ & $22.50 \pm 0.25 \mathrm{bcd}$ & $4.07 \pm 1.04 \mathrm{de}$ \\
\hline & & $\begin{array}{l}\text { Cúpulas } \\
\text { insertadas }\end{array}$ & $2.35 \pm 0.62 \mathrm{~cd}$ & $2.66 \pm 0.20$ ef & $7.51 \pm 0.02 b c$ & $23.17 \pm 0.11 \mathrm{~b}$ & $7.35 \pm 0.91 \mathrm{abc}$ \\
\hline & & Brazos colectores & $2.66 \pm 0.42 \mathrm{abc}$ & $1.97 \pm 0.14 \mathrm{f}$ & $7.42 \pm 0.01 \mathrm{~cd}$ & $23.11 \pm 0.08 \mathrm{bc}$ & $5.91 \pm 0.31 \mathrm{~cd}$ \\
\hline \multirow[t]{3}{*}{0.30} & 60 & Medio poroso & $2.58 \pm 0.48 \mathrm{abc}$ & $3.23 \pm 0.12$ bcde & $7.39 \pm 0.01 \mathrm{de}$ & $21.46 \pm 0.22$ cde & $6.29 \pm 0.23 \mathrm{bc}$ \\
\hline & & $\begin{array}{l}\text { Cúpulas } \\
\text { insertadas }\end{array}$ & $2.38 \pm 0.41 \mathrm{~cd}$ & $3.45 \pm 0.13 \mathrm{bcd}$ & $7.44 \pm 0.01 \mathrm{bcd}$ & $20.04 \pm 0.17$ ef & $5.77 \pm 0.17 \mathrm{~cd}$ \\
\hline & & Brazos colectores & $2.43 \pm 0.49 \mathrm{~cd}$ & $3.79 \pm 0.10 \mathrm{abc}$ & $7.38 \pm 0.01 \mathrm{de}$ & $19.37 \pm 0.27 \mathrm{f}$ & $5.98 \pm 0.24 \mathrm{~cd}$ \\
\hline
\end{tabular}

Debido a la heterogeneidad del efluente utilizado a lo largo del ensayo, se observaron diferencias significativas para un mismo parámetro entre condiciones operativas y diseño del filtro. Sin embargo, no hubo diferencias significativas en las condiciones de $0.20 \mathrm{~m}$ de altura de lecho filtrante y $30 \mathrm{~m} / \mathrm{h}$ de velocidad de filtración, ni en las de $0.30 \mathrm{~m}$ y $60 \mathrm{~m} / \mathrm{h}$, salvo en la temperatura en esta última condición.

\subsection{Tratamiento de datos y análisis estadístico}

Los datos de tiempo de filtración, caudal y volumen de filtración y lavado, presión a la entrada y salida del filtro, parámetros fisicoquímicos a la entrada y salida de los filtros, consumo eléctrico de filtración y lavado e inyección de cloro fueron medidos y registrados cada minuto por un sistema SCADA desarrollado previamente [16] y adaptado al presente ensayo.

La eficiencia de reducción (E) de turbidez y oxígeno disuelto se calculó con la expresión:

$$
E=\frac{N o-N}{N o} \times 100
$$




\section{CONGRESO IBÉRICO DE AGROINGENIERÍA \\ X CONGRESSO IBÉRICO DE AGROENGENHARIA \\ 3 - 6 septiembre 2019, Huesca - España}

donde $\mathrm{N}_{0}$ y $\mathrm{N}$ son los valores de dichos parámetros a la entrada y salida de los filtros, respectivamente.

El volumen filtrado por unidad de consumo de energía eléctrica $\left(\mathrm{m}^{3} / \mathrm{kWh}\right)$ fue calculado con la fórmula:

$$
V e e c=\frac{V f}{E E f+E E b}
$$

donde $\mathrm{V}_{\mathrm{f}}$ es el volumen filtrado de cada ciclo de filtración $\left(\mathrm{m}^{3}\right)$ y EEf y EEb el consumo eléctrico durante un ciclo de filtración y lavado, respectivamente $(\mathrm{kWh})$.

Se consideró un ciclo completo el tiempo comprendido entre dos lavados (desde el inicio de un lavado hasta terminar el proceso de filtración al llegar a una pérdida de carga de $50 \mathrm{kPa}$ entre la presión inicial y final del filtro). Todos aquellos ciclos en los que no se alcanzaron los $50 \mathrm{kPa}$ de caída de presión o en los que la toma de datos no fue fiable (debido, por ejemplo, a sensores descalibrados, realización de operaciones de mantenimiento, o con lavados ineficientes) se excluyeron del análisis de datos.

Para el análisis estadístico se utilizó el programa SPSS Statistics (IBM, New York, Estados Unidos). Para cada parámetro, el modelo utilizado incluyó como parámetros fijos el diseño del drenaje, la altura del medio filtrante y la velocidad de filtración. La turbidez de entrada fue tomada como covariable en el modelo cuando ésta tuvo efecto significativo. Para la diferenciación de medias, se utilizó el test de Tukey con un intervalo de confianza del 95\%.

\section{Resultados y discusión}

\subsection{Caracterización del volumen de agua y energía eléctrica consumida}

La Tabla 3 muestra los valores medios del volumen de filtración y lavado, la ratio entre el volumen de contralavado y el volumen total, el consumo de energía eléctrica durante la filtración y contralavado, la ratio entre el consumo eléctrico de contralavado respecto el total y la ratio entre el volumen de agua en el proceso de filtración respecto al consumo de energía eléctrica total.

Tabla 3. Medias y error estándar de los volúmenes de agua y consumo de energía eléctrica para cada condición operativa.

\begin{tabular}{|c|c|c|c|c|c|c|c|c|c|}
\hline $\begin{array}{c}\begin{array}{c}\text { Altura } \\
\text { de } \\
\text { lecho }\end{array} \\
(\mathrm{m})\end{array}$ & $\begin{array}{c}\text { Velocidad } \\
\text { de } \\
\text { filtración }\end{array}$ & $\begin{array}{l}\begin{array}{l}\text { Diseño del } \\
\text { drenaje }\end{array} \\
(\mathrm{m} / \mathrm{h})\end{array}$ & $\begin{array}{l}\text { Volumen } \\
\text { filtrado por } \\
\text { ciclo de } \\
\text { filtración } \\
\left(\mathrm{m}^{3}\right)\end{array}$ & $\begin{array}{c}\text { Volumen de } \\
\text { contralavado } \\
\text { por ciclo de } \\
\text { filtración } \\
\left(\mathrm{m}^{3}\right)\end{array}$ & $\begin{array}{c}\text { Ratio volumen } \\
\text { lavado/Total ciclo } \\
\text { (\%) }\end{array}$ & $\begin{array}{c}\text { Consumo } \\
\text { eléctrico } \\
\text { filtración } \\
\text { (kWh) }\end{array}$ & $\begin{array}{c}\begin{array}{c}\text { Consumo } \\
\text { eléctrico } \\
\text { contralavado }\end{array} \\
(\mathrm{kWh})\end{array}$ & $\begin{array}{c}\text { Ratio consumo } \\
\text { eléctrico } \\
\text { contralavado/total } \\
\text { (\%) }\end{array}$ & $\begin{array}{l}\text { Volumen } \\
\text { filtrado/Consumo } \\
\text { eléctrico total } \\
\text { ciclo } \\
\left(\mathrm{m}^{3} / \mathrm{kWh}\right)\end{array}$ \\
\hline \multirow[t]{3}{*}{0.20} & 30 & Medio poroso & $31.04 \pm 4.84$ & $0.45 \pm 0.003$ & $1.76 \pm 0.27$ & $3.14 \pm 0.41$ & $0.02 \pm 0.001$ & $0.78 \pm 0.11$ & $8.70 \pm 0.04$ \\
\hline & & $\begin{array}{l}\text { Cúpulas } \\
\text { insertadas }\end{array}$ & $17.51 \pm 1.64$ & $0.45 \pm 0.003$ & $3.21 \pm 0.35$ & $2.07 \pm 0.19$ & $0.02 \pm 0.001$ & $1.12 \pm 0.12$ & $8.35 \pm 0.10$ \\
\hline & & $\begin{array}{c}\text { Brazos } \\
\text { colectores }\end{array}$ & $15.73 \pm 1.02$ & $0.46 \pm 0.002$ & $3.54 \pm 0.29$ & $1.84 \pm 0.12$ & $0.02 \pm 0.001$ & $1.17 \pm 0.12$ & $8.48 \pm 0.03$ \\
\hline \multirow[t]{3}{*}{0.20} & 60 & Medio poroso & $36.43 \pm 3.30$ & $0.77 \pm 0.003$ & $5.14 \pm 1.17$ & $4.18 \pm 0.39$ & $0.04 \pm 0.001$ & $2.94 \pm 0.94$ & $8.51 \pm 0.10$ \\
\hline & & $\begin{array}{l}\text { Cúpulas } \\
\text { insertadas }\end{array}$ & $54.19 \pm 5.26$ & $0.77 \pm 0.007$ & $2.37 \pm 0.39$ & $6.17 \pm 0.60$ & $0.04 \pm 0.001$ & $0.96 \pm 0.15$ & $8.70 \pm 0.07$ \\
\hline & & $\begin{array}{l}\text { Brazos } \\
\text { colectores }\end{array}$ & $55.45 \pm 8.50$ & $0.77 \pm 0.005$ & $3.72 \pm 0.71$ & $6.51 \pm 1.01$ & $0.04 \pm 0.001$ & $2.01 \pm 0.43$ & $8.56 \pm 0.05$ \\
\hline \multirow[t]{3}{*}{0.30} & 30 & Medio poroso & $92.12 \pm 21.53$ & $0.45 \pm 0.002$ & $1.76 \pm 1.18$ & $10.76 \pm 2.50$ & $0.01 \pm 0.002$ & $0.40 \pm 0.24$ & $8.50 \pm 0.05$ \\
\hline & & $\begin{array}{l}\text { Cúpulas } \\
\text { insertadas }\end{array}$ & $39.82 \pm 4.89$ & $0.45 \pm 0.002$ & $2.60 \pm 0.53$ & $4.81 \pm 0.59$ & $0.02 \pm 0.001$ & $0.90 \pm 0.20$ & $8.22 \pm 0.04$ \\
\hline & & $\begin{array}{c}\text { Brazos } \\
\text { colectores }\end{array}$ & $37.14 \pm 3.95$ & $0.46 \pm 0.002$ & $2.23 \pm 0.46$ & $4.59 \pm 0.49$ & $0.02 \pm 0.001$ & $0.85 \pm 0.21$ & $8.11 \pm 0.13$ \\
\hline \multirow[t]{3}{*}{0.30} & 60 & Medio poroso & $33.10 \pm 2.57$ & $0.68 \pm 0.017$ & $2.97 \pm 0.27$ & $4.08 \pm 0.32$ & $0.04 \pm 0.001$ & $1.40 \pm 0.10$ & $8.01 \pm 0.10$ \\
\hline & & $\begin{array}{l}\text { Cúpulas } \\
\text { insertadas }\end{array}$ & $24.22 \pm 1.40$ & $0.75 \pm 0.008$ & $4.14 \pm 0.23$ & $3.00 \pm 0.17$ & $0.05 \pm 0.001$ & $2.15 \pm 0.13$ & $7.94 \pm 0.04$ \\
\hline & & $\begin{array}{c}\text { Brazos } \\
\text { colectores }\end{array}$ & $33.66 \pm 1.60$ & $0.76 \pm 0.006$ & $2.76 \pm 0.17$ & $4.38 \pm 0.21$ & $0.04 \pm 0.001$ & $1.27 \pm 0.08$ & $7.67 \pm 0.06$ \\
\hline
\end{tabular}




\section{CONGRESO IBÉRICO DE AGROINGENIERÍA \\ X CONGRESSO IBÉRICO DE AGROENGENHARIA \\ 3 - 6 septiembre 2019, Huesca - España}

A velocidades de filtración altas $(60 \mathrm{~m} / \mathrm{h})$ se filtró más volumen de agua y se consumió más energía eléctrica, excepto en las condiciones de $0.30 \mathrm{~m}$ y $60 \mathrm{~m} / \mathrm{h}$, donde los ciclos fueron cortos debido a que alcanzaban la consigna de pérdida de carga rápidamente. En general, con alturas de lecho filtrante de $0.20 \mathrm{~m}$ se filtró más volumen de agua por unidad de energía eléctrica consumida que con alturas de $0.30 \mathrm{~m}$.

\subsection{Efecto del diseño del drenaje y las condiciones operativas en la calidad del efluente}

Las eficiencias de reducción del oxígeno disuelto (OD) y turbidez se calcularon mediante la ecuación (1) y fueron tratadas estadísticamente. Se halló un efecto significativo de la interacción entre el diseño del drenaje y la velocidad de filtración $(\mathrm{P}<0.05)$ en la reducción del oxígeno disuelto y una triple interacción de los tres factores en la reducción de turbidez $(\mathrm{P}<0.001)$.

Bajo velocidades de $30 \mathrm{~m} / \mathrm{h}$ no hubo diferencias significativas $(\mathrm{P}<0.05)$ entre diseños de drenajes en la variación de oxígeno disuelto, aunque el diseño de brazos colectores obtuvo aumentos mayores. Sin embargo, para velocidades de $60 \mathrm{~m} / \mathrm{h}$, el incremento de OD fue significativamente mayor en el diseño de cúpulas insertadas (31.10\%) que en el de medio poroso $(11.22 \%)$ y el de brazos colectores $(-3.52 \%)$.

En la reducción de turbidez, se detectó una interacción triple de los factores diseño del drenaje, altura del lecho filtrante y velocidad de filtración. Estos dos últimos factores fueron estudiados para cada diseño del drenaje (Figura 3).

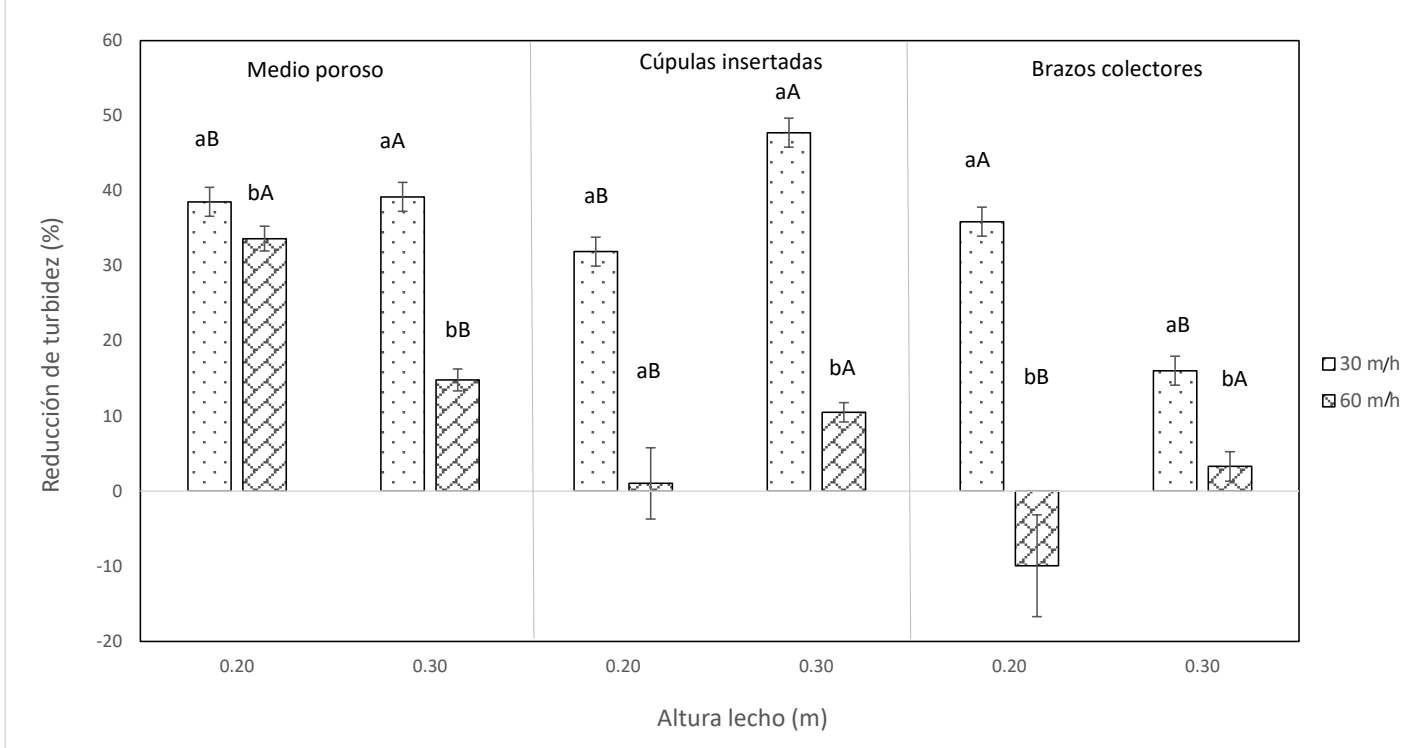

Figura 3. Interacciones entre altura del lecho filtrante y velocidades de filtración para cada diseño del drenaje, en los valores de reducción de turbidez. Para cada altura y diseño, letras minúsculas diferentes indican diferencias significativas $(\mathrm{P}<0.05)$ entre velocidades. Para cada velocidad y diseño, diferentes mayúsculas distintas indican diferencias significativas $(\mathrm{P}<0.05)$ entre alturas de lecho filtrante.

En general, el filtro con drenaje de medio poroso presentó una reducción mayor de la turbidez que los otros dos filtros para todas las condiciones operativas, excepto para una altura de lecho de $0.30 \mathrm{~m}$ y $30 \mathrm{~m} / \mathrm{h}$ de velocidad de filtración, donde fue el diseño de cúpulas insertadas el que obtuvo una reducción mayor ( $47.74 \%$ respecto $39.19 \%$ ). Para todos los diseños de drenajes, velocidades altas de filtración $(60 \mathrm{~m} / \mathrm{h})$ presentaron reducciones de turbidez menores que con velocidades bajas $(30 \mathrm{~m} / \mathrm{h})$. Sin embargo, no hubo un patrón de comportamiento tan claro por lo que a alturas de lecho filtrante se refiere. Algunos autores han observado reducciones mayores 


\section{CONGRESO IBÉRICO DE AGROINGENIERÍA \\ X CONGRESSO IBÉRICO DE AGROENGENHARIA \\ 3 - 6 septiembre 2019, Huesca - España}

de turbidez con velocidades bajas de filtración [17, 18], que con velocidades altas. Las reducciones de turbidez tienden a producirse en las primeras capas del lecho filtrante mientras que con velocidades inferiores este fenómeno no es tan evidente [18]. El diseño del drenaje también afecta al proceso de contralavado, que puede estar relacionado con la reducción de turbidez, ya que los drenajes son esenciales para garantizar la eliminación de las partículas retenidas durante el lavado [19].

\subsection{Efecto del diseño del drenaje y las condiciones operativas en el consumo de agua y energía eléctrica.}

El análisis de los datos de volumen utilizado en el proceso de filtración por unidad de energía eléctrica total utilizada en un ciclo de filtración halló interacciones significativas $(\mathrm{P}<0.001)$ entre los factores de altura del lecho filtrante y velocidad de filtración, y los de diseño del drenaje y velocidad de filtración $(\mathrm{P}<0.001)$.

En lo que a la interacción entre altura del lecho filtrante y velocidad de filtración se refiere, el volumen de agua filtrado por unidad de energía eléctrica consumida, bajo una misma velocidad, fue significativamente mayor con alturas de 0.20 que de $0.30 \mathrm{~m}$ (8.47 frente 8.21 $\mathrm{m}^{3} / \mathrm{kWh}$, respectivamente para $30 \mathrm{~m} / \mathrm{h}$; y 8.57 frente $7.87 \mathrm{~m} 3 / \mathrm{kWh}$, respectivamente para $60 \mathrm{~m} / \mathrm{h}$ ). Sin embargo, no se encontraron diferencias entre velocidades para la altura de lecho de $0.20 \mathrm{~m}$, pero sí para la altura de $0.30 \mathrm{~m}$, siendo la ratio mayor con la velocidad de $30 \mathrm{~m} / \mathrm{h}$ que con la de 60 $\mathrm{m} / \mathrm{h}\left(8.21\right.$ ante $\left.7.87 \mathrm{~m}^{3} / \mathrm{kWh}\right)$.

En la Figura 4 se muestra la interacción entre la velocidad de filtración y el diseño del drenaje. Para velocidades de $30 \mathrm{~m} / \mathrm{h}$, el diseño de medio poroso filtró significativamente $(\mathrm{P}<0.05)$ más volumen de agua por unidad de energía eléctrica consumida $\left(8.61 \mathrm{~m}^{3} / \mathrm{kWh}\right)$ que el diseño de brazos colectores y el de cúpulas insertadas $\left(8.33\right.$ y $8.27 \mathrm{~m}^{3} / \mathrm{kWh}$, respectivamente). Para velocidades de $60 \mathrm{~m} / \mathrm{h}$, los diseños de medio poroso y cúpulas insertadas presentaron una ratio superior $\left(8.25\right.$ y $8.14 \mathrm{~m}^{3} / \mathrm{kWh}$, respectivamente) que el diseño de brazos colectores $\left(7.88 \mathrm{~m}^{3} / \mathrm{kWh}\right)$. Tanto el diseño de medio poroso como el de brazos colectores tuvieron una ratio mayor con velocidades de filtración de $30 \mathrm{~m} / \mathrm{h}$ que con $60 \mathrm{~m} / \mathrm{h}\left(8.61\right.$ frente $8.25 \mathrm{~m}^{3} / \mathrm{kWh}$ para el medio poroso y 8.33 frente $7.88 \mathrm{~m}^{3} / \mathrm{kWh}$ para el de brazos colectores).

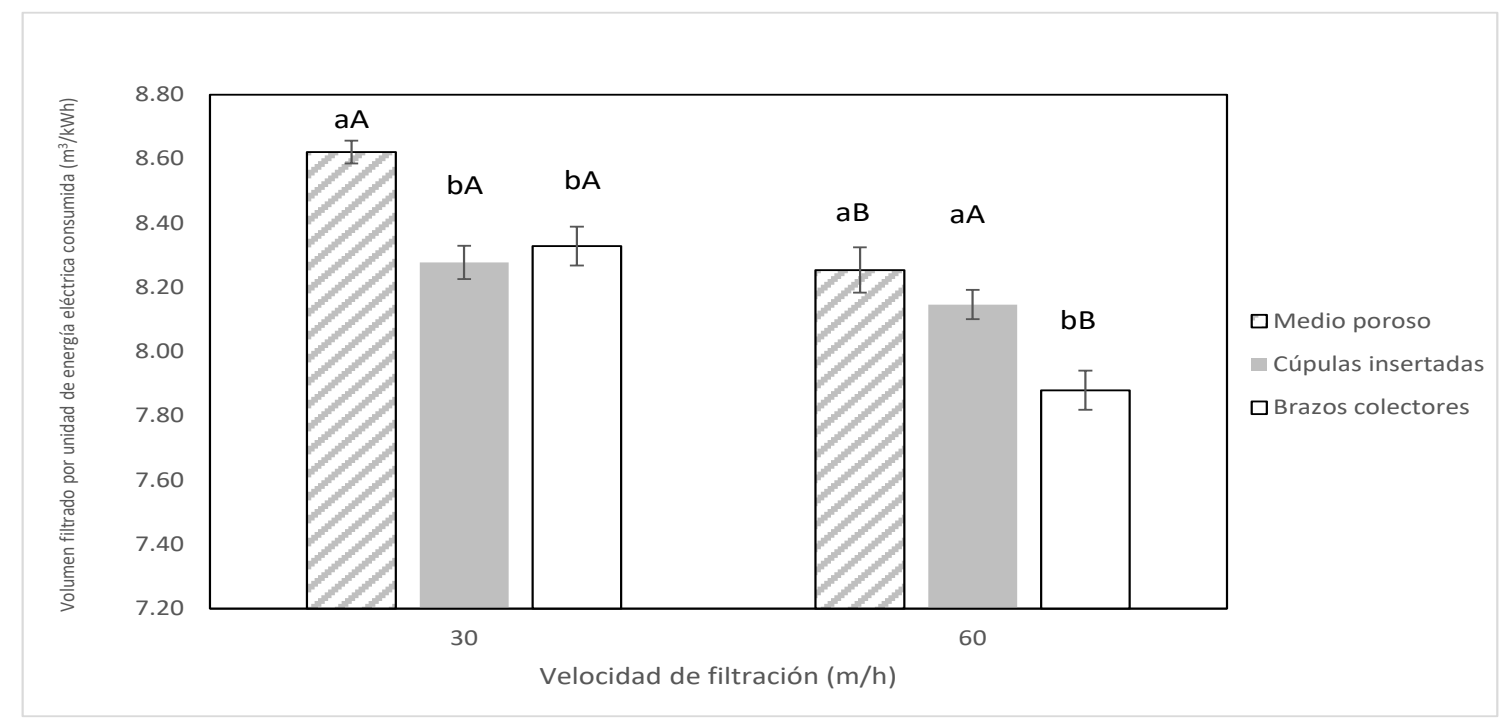

Figura 4. Interacciones entre velocidades de filtración y diseño del drenaje en el volumen de agua filtrada por unidad de consumo de energía eléctrica $\left(\mathrm{m}^{3} / \mathrm{kWh}\right)$. Para cada velocidad de filtración, letras minúsculas diferentes indican diferencias significativas $(\mathrm{P}<0.05)$ entre diseños de drenajes. Para cada diseño de drenaje, letras mayúsculas diferentes indican diferencias significativas $(\mathrm{P}<0.05)$ entre velocidades de filtración. 


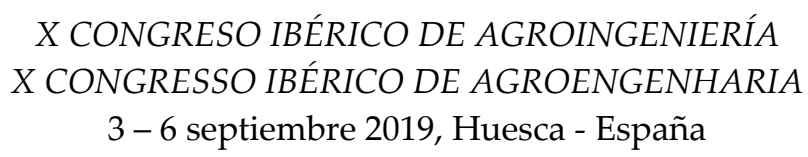

Finalmente, los resultados del volumen utilizado en filtración por unidad de consumo de energía eléctrica concuerdan con los obtenidos en otros estudios [17], en donde el diseño de tres filtros de arena, diferentes condiciones operativas y el tamaño de arena utilizada como medio, tuvieron efecto en la caída de presión dentro del filtro. En dicho estudio, la caída de presión incrementó tanto para velocidades de filtración como para alturas de lecho mayores en todos los filtros testados, que fueron de características similares a los del presente ensayo.

\section{Conclusiones}

Las condiciones operativas y los distintos diseños del drenaje afectan a la calidad del efluente, al volumen de agua filtrada y al consumo energético eléctrico en los filtros de arena utilizados en sistemas de riego por goteo utilizando aguas residuales.

Se observó la existencia de una interacción triple entre el diseño del drenaje, la altura del lecho filtrante y la velocidad de filtración en la reducción de la turbidez conseguida por los filtros. Cada filtro presentó mayores reducciones de la turbidez a una velocidad de filtración de $30 \mathrm{~m} / \mathrm{h}$. Asimismo, el diseño del drenaje de medio poroso presentó reducciones de turbidez mayores que los otros dos diseños para todas las condiciones operativas ensayadas, excepto para la altura de lecho de $0.30 \mathrm{~m}$ y velocidad de filtración de $30 \mathrm{~m} / \mathrm{h}$, donde los niveles de entrada de turbidez para este diseño fueron muy bajos comparados con los que tuvieron los otros dos filtros.

Por otro lado, se filtró mayor volumen de agua por unidad de energía eléctrica consumida con una altura de lecho de 0.20 que con $0.30 \mathrm{~m}\left(8.53\right.$ frente $\left.7.95 \mathrm{~m}^{3} / \mathrm{kWh}\right)$, y bajo esta última altura, también fue mayor la ratio bajo $30 \mathrm{~m} / \mathrm{h}$ que con $60 \mathrm{~m} / \mathrm{h}(8.21$ frente $7.87 \mathrm{~m} / \mathrm{kWh})$. El diseño de drenaje de medio poroso presentó valores significativamente superiores que los otros dos diseños para ambas velocidades de filtración, especialmente a una velocidad de filtrado de $30 \mathrm{~m} / \mathrm{h}$.

Finalmente, cuando se utilicen aguas residuales regeneradas con características similares a las de este ensayo, velocidades bajas de filtración proporcionan mayores reducciones de turbidez $(34.17 \%$ con $30 \mathrm{~m} / \mathrm{h}$ respecto $11.27 \%$ con $60 \mathrm{~m} / \mathrm{h}$ ) y más volumen de agua filtrada por unidad de energía eléctrica consumida ( 8.35 respecto $8.11 \mathrm{~m}^{3} / \mathrm{kWh}$ ). Asimismo, el diseño de medio poroso propuesto por Bové et al. (2017) [14] obtuvo una reducción media de turbidez superior (12.83\%) que los otros dos diseños estudiados, ofreciendo una mayor protección a la obturación de los goteros, además de consumir un $2.77 \%$ menos de energía eléctrica respecto al diseño que mostró los valores más altos de consumo energético. La altura del lecho filtrante no mostró un patrón claro en cuanto a la reducción de turbidez, aunque alturas menores permitieron filtrar más volumen de agua por unidad de energía eléctrica consumida.

\section{Agradecimientos}

Los autores quieren expresar su agradecimiento al Ministerio de Economía y Competitividad por financiar el presente ensayo, incluido en el proyecto de investigación con referencia AGL2015-63750-R. Carles SoléTorres es beneficiario de la beca pre-doctoral IFUdG2016/72 otorgada por la Universidad de Girona. Los autores también quieren agradecer al Municipio de Celrà su ayuda en la realización de este experimento.

\section{Referencias}

1. Asano T., Burton F.L., Leverenz H.L. Water reuse: issues, technologies and applications. 2007, McGraw Hill Inc, New York.

2. Bucks D.A., Nakayama F.S., Gilbert R.G. Trickle irrigation water quality and preventive maintenance. Agric. Water Manage. 1979, 2 (2), 149-162.

3. Ravina I., Paz E., Sofer Z., Marcu A., Shisha A., Sagi G. Control of emitter clogging in drip irrigation with reclaimed wastewater. Irrig. Sci. 1992, 13 (3), 129-139.

4. Oron G., Shelef G., Turzynski B. Trickle irrigation using treated wastewaters. J. Irrig. Drain. Div., 1979, 105 (IR2), 175-186. 


\section{CONGRESO IBÉRICO DE AGROINGENIERÍA \\ X CONGRESSO IBÉRICO DE AGROENGENHARIA \\ 3 - 6 septiembre 2019, Huesca - España}

5. Capra A., Scicolone B. Recycling of poor quality urban wastewater by drip irrigation systems. J. Clean Prod. 2007, 15 (16), 1529-1534.

6. Duran-Ros M., Puig-Bargués J., Arbat G., Barragán J., Ramírez de Cartagena F. Effect of filter, emitter and location on clogging when using effluents. Agric. Water Manage. 2009, 96 (10), 67-79.

7. Tajrishy M.A., Hills D.J., Tchobanoglous G. Pretreatment of secondary effluent for drip irrigation. J. Irrig. Drain. Eng. 1994, 120 (4), 716-731.

8. Trooien T.P., Hills D.J. Application of biological effluent. In: Lamm, F.R., Ayars, J.E., Nakayama, F.S. (Eds.), Microirrigation for Crop Production. Design, Operation, and Management. 2007, Elsevier, Amsterdam, pp. 329-353.

9. Puig-Bargués J., Barragán J., Ramírez de Cartagena, F. Filtration of effluents for microirrigation systems. Trans. ASAE. 2005, 48(3): 968-978.

10. Bové J., Arbat G., Pujol T., Duran-Ros M., Ramírez de Cartagena F., Velayos J., Puig-Bargués J. Reducing energy requirements for sand filtration in microirrigation: improving the underdrain and packing. Biosyst. Eng. 2015, 140, 67-78.

11. Tarjuelo J.M., Rodríguez-Díaz J.A., Abadía R., Camacho E., Rocamora C., Moreno M.A. Efficient water and energy use in irrigation modernization: Lessons from Spanish case studies. Agric. Water Manage. 2015, 162, 67-77.

12. Burt C., Howes D. J., Freeman B. Public Interest Energy Research (PIER) Program. Final Project Report, Agriculture Water Energy Efficiency. 2011, California Energy Commission and Irrigation Training and Research Center, San Luis Obispo, California, 263 pp.

13. Arbat G., Pujol T., Puig-Bargués J., Duran-Ros M., Barragán J., Montoro L., Ramírez de Cartagena F. Using computational fluid dynamics to predict head losses in the auxiliary elements of a microirrigation sand filter. Trans. ASABE. 2011, 54(4), 1367 -1376.

14. Bové J., Puig-Bargués J., Arbat G., Duran-Ros M., Pujol T., Pujol J., Ramírez de Cartagena F. Development of a new underdrain for improving the efficiency of microirrigation sand media filters. Agric. Water Manage. 2017, 179, 296-305.

15. Bové J., Pujol J., Arbat G., Duran-Ros M., Ramírez de Cartagena F., Puig-Bargués J. Environmental assessment of underdrain designs for a sand media filter. Biosyst. Eng. 2018, 167, 126-136.

16. Duran-Ros M., Puig-Bargués J., Arbat G., Barragán J., Ramírez de Cartagena F. Definition of a SCADA system for a microirrigation network with effluents. Comput. Electron. Agric. 2008, 64 (2), 338-342.

17. Mesquita M., Testezlaf R., Ramirez J. The effect of media bed characteristics and internal auxiliary elements on sand filter head loss. Agric. Water Manage. 2012, 115, 178-185.

18. De Deus F.P., Testezlaf R., Mesquita M. Assessment methodology of backwashing in pressurized sand filters. Rev. Bras. Eng. Agr. Amb. 2016, 20 (7), 600-605.

19. Mesquita M. Desenvolvimento tecnológico de um filtro de areia para irrigação localizada. Tese de Doutorado. 2014, FEAGRI/UNICAMP, Campinas, 169 pp. 